function, $44 \%$ of the subjects had some degree of dysfunction. The rest of the variables are shown in table 1.

Conclusions Sexual function is affected in men with lupus (mostly young and with adequate functional capacity), regardless of comorbidities and treatment. Interestingly, lymphopenia is persistently associated with an impaired sexual function, which could be related to the role it plays in endothelial dysfunction and atherosclerosis. The patients disease perception, which is influenced by their academic level and physical role in their daily activities, seems to affect their sexual performance and quality of life. These findings reinforce the need of a multidisciplinary approach for male SLE patients with sexual dysfunction.

Funding Source(s): None

\section{INCIDENCE AND RISK FACTORS FOR PROGRESSION OF CORONARY-ARTERY CALCIFICATION IN PATIENTS WITH SYSTEMIC LUPUS ERYTHEMATOSUS (SLE)}

${ }^{1}$ Mario Cesar Ocampo-Torres, ${ }^{1}$ Gabriela Hernandez-Molina, ${ }^{2}$ Sergio Criales, ${ }^{1}$ Juanita Romero-Diaz*. ${ }^{1}$ Instituto Nacional de Ciencias Médicas y Nutrición S.Z; ${ }^{2}$ Instituto Nacional de Cardiologia Ignacio Chavez

10.1136/lupus-2019-Ism.153

Background Premature atherosclerosis has been recognized as a major cause of morbidity and mortality in SLE patients. Whether the effect of risk factors for cardiovascular disease in patients with lupus change over time is still unknown. We aimed to identify the incidence and risk factors for progression of coronary-artery calcification (CAC) in SLE patients.

Methods Design: Inception Cohort. Since enrollment into the cohort, all patients had a standardized medical history, physical examination, and laboratory tests, including lipid profile, apoB, homocystein, high-sensitivity C-reactive protein (hs$\mathrm{CRP}$ ), serum complement (C3 and $\mathrm{C} 4$ ), and autoantibodies. Every 3-6 months, patients have been seen at the lupus clinic for medical care, and assessments of disease activity using the SLE disease activity scores, and medications usage.
Every year, information has been updated, including irreversible damage accrual, any co-morbidities, traditional cardiovascular risk-factors, and a blood sample has been drawn. In 2008, 104 lupus patients from the cohort (93\% females) was screened for coronary-artery calcifications using Multidetector Computed Tomography, after 5.1 years of follow-up. In 2018 a follow-up screening for CAC was carried-up. CAC was considered as positive if i) patients without $\mathrm{CAC}$ in 2008 were found with CAC + in the second screening or ii) patients with CAC positive in 2008 were found with any increase of their Calcium Score. Correlates for calcifications were analyzed. Cumulative incidence of CAC was calculated and risk factors for CAC progression were identified by multivariate analysis.

Results At-enrollment into de cohort, lupus patients were 27.2 +9.1 years of age and disease duration $5.4+3.8$ months. On 2008 during the first screening, coronary-artery calcification were detected in $7.2 \%$ patients, since age 23 years, and from three years of diagnosis. At follow-up screening, progression of CAC was identified in 16.3\% (IC95\% 10.4-24.6). Cumulative incidence of CAC was observed in 9\%. Earlier Risk factors associated with CAC were disease activity $(p=0.03)$ and disease duration $(p=0.03)$ while risk factors for progression of CAC were postmenopausal status $(p=0.01)$, apoB levels $(\mathrm{p}=0.01)$.

Conclusions Our findings suggest that in patients with SLE earlier CAC is associated with disease severity while in the progression of CAC, traditional risk factors for atherosclerosis were adding.

Funding Source(s): None

\section{IMPACT OF DIAGNOSIS AGE ON QUALITY OF LIFE AMONG PATIENTS WITH SYSTEMIC LUPUS ERYTHEMATOSUS}

${ }^{1}$ Christina L Kearse* ${ }^{2}$ Robert Campbell, ${ }^{3}$ Jim Oates, ${ }^{3}$ Gary S Gilkeson, ${ }^{3}$ Diane L Kamen. ${ }^{1}$ College of Medicine, Medical University of South Carolina; ${ }^{2}$ Center for Health Analytics and Discovery, Eastern Virginia Medical School; ${ }^{3}$ Division of Rheumatology and Immunology, Medical University of South Carolina

10.1136/lupus-2019-Ism.154

\begin{tabular}{|c|c|c|c|}
\hline Domain & $\begin{array}{l}\operatorname{aSLE}(n=323) \\
\text { Mean } \pm S D\end{array}$ & $\begin{array}{l}\text { cSLE }(n=9) \\
\text { Mean } \pm \text { SD }\end{array}$ & P Value \\
\hline \multicolumn{4}{|l|}{ Physical Health } \\
\hline Physical Functioning & $38.8 \pm 12.4$ & $52.1 \pm 6.1$ & $0.002^{*}$ \\
\hline Role Physical & $38.5 \pm 12.5$ & $50.1 \pm 7.1$ & $0.007^{*}$ \\
\hline Bodily Pain & $41.8 \pm 11.3$ & $48.0 \pm 11.3$ & 0.103 \\
\hline General Health & $37.7 \pm 11.3$ & $41.7 \pm 7.9$ & 0.289 \\
\hline \multicolumn{4}{|l|}{ Mental Health } \\
\hline Vitality & $43.1 \pm 11.6$ & $50.4 \pm 8.6$ & 0.064 \\
\hline Social Functioning & $40.7 \pm 12.7$ & $46.5 \pm 8.3$ & 0.172 \\
\hline Role Emotional & $41.0 \pm 14.9$ & $48.5 \pm 8.1$ & 0.133 \\
\hline Mental Health & $45.3 \pm 13.5$ & $48.8 \pm 12.5$ & 0.442 \\
\hline
\end{tabular}

